\section{Evaluation of Laboratory Findings, Clinical Features and Rates of Diagnosis of Patients Admitted to Outpatient Clinic of Pediatric Neurology with Neuromuscular Manifestations}

\author{
Çocuk Nöroloji Polikliniğine Nöromüsküler Belirtilerle \\ Başvuran Hastaların Laboratuvar Bulgularının, Klinik \\ Özelliklerinin ve Tanı Oranlarının Değerlendirilmesi
}

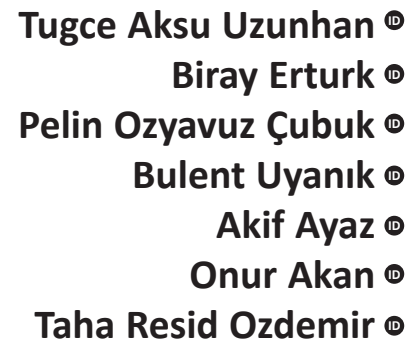

\section{ABSTRACT}

Objective: Our aim is to evaluate how many patients with neuromuscular manifestations get a definite diagnosis and which methods are used in the pathway to diagnosis as well as to assess patient characteristics.

Methods: Patients aged 0-18 years old with neuromuscular manifestations (e.g., weakness, hypotonia, creatine kinase elevation) who were admitted to Okmeydani Training and Research Hospital between January 2017 and July 2019 were included. Retrospectively, patient demographics, clinical signs, laboratory tests, diagnoses, clinical follow-up were recorded.

Results: Forty-five patients aged $67.8 \pm 59.6$ months were included in the study. Thirteen (29\%) patients were female, and $32(71 \%)$ were male. Creatine kinase levels were increased in $26(58 \%)$ patients (median: $3211 \mathrm{IU} / \mathrm{L}$ ). Twenty-four patients underwent electromyography; seven patients had neuropathy and nine patients muscular pathologies. Three (0.07\%) patients underwent muscle biopsy and had nonspecific myopathic changes. Twenty-six (58\%) patients out of 45 had a definite diagnosis, and 21 of these diagnoses were genetically confirmed. Seven patients had been subjected to next generation sequencing, and five of these were diagnosed with dystrophinopathy, hypokalemic periodic paralysis, mental retardation autosomal dominant type 9, Ullrich muscular dystrophy, and calpainopathy. Altogether, the most common diagnoses were dystrophinopathy, spinal muscular atrophy, and chronic inflammatory demyelinating polyneuropathy.

Conclusion: After a patient history is taken, a physical examination is conducted, and serum creatine kinase levels are measured, establishment of diagnosis is possible through targeted genetic tests for diseases like dystrophinopathy. However, for patients who cannot be diagnosed with this approach, neuromuscular panels and whole exome sequencing can provide a diagnosis.

Keywords: Creatine kinase, neuromuscular manifestations, next-generation sequencing, Duchenne muscular dystrophy, whole exome sequencing

öz

Amaç: Bu çalışmada amacımız, nöromüsküler hastalıkları düşündüren semptom ve bulgularla başvuran çocuk hastaların ne kadarının kesin tanı aldığının, bu hastalarda hangi yöntemler ile tanıya ulaşıldığının ve hasta özelliklerinin değerlendirilmesidir.

Yöntem: Okmeydanı Eğitim ve Araştırma Hastanesi Çocuk Nöroloji Polikliniği'ne Ocak 2017-Temmuz 2019 tarihleri arasında nöromüsküler belirtilerle (örn: güçsüzlük, hipotoni, kreatin kinaz yüksekliği) başvuran 0-18 yaş arası hastalar değerlendirmeye alındı. Retrospektif olarak hastaların demografik verileri, klinik bulguları, laboratuvar testleri, tanıları, izlemleri kayıt altına alındı.

Bulgular: Çalışmaya dahil edilen 45 hastanın yaş ortalaması 67,8 $\$ 59,6$ ay idi. On üç (\%29)'ü kız, 32 (\%71)'sierkekti. Kreatin kinaz seviyeleri 26(\%61) hastada yüksekti ve medyanı 3211 IU/L idi. Elektromiyografi yapılan 24 hastadan yedisinde nöropati, dokuz hastada kasa ait patolojiler saptandı. Kas biyopsisi yapılan üç $(\% 0,07)$ hastada non spesifik miyopatik değişiklikler izlendi. Kırkbeş hastadan 26 (\%58)'sına kesin tanı konulabildi, bunlardan 21 (\%47)'inin tanısı genetik olarak kesinleştirildi. Yedi hastada yeni nesil dizileme analizi uygulandı ve beşi distrofinopati, hipokalemik periyodik paralizi, otozomal dominant mental retardasyon tip 9, Ullrich müsküler distrofi, calpainopati tanılarını aldı. Tüm hastalarda en sık tanılar distrofinopati, spinal müsküler atrofi ve kronik inflamatuvar demiyelinizan polinöropatiydi.

Sonuç: Öykü, fizik muayene ve serum kreatin kinaz seviyesi sonrasında, örneğin distrofinopati gibi bir grup hastalıkta hedefe yönelik genetik testler ile tanıya ulaşılabilir. Ancak bu şekilde tanıya ulassılamayan hastalarda nöromüsküler hastalık panelleri ve tüm ekzom dizileme gibi ileri moleküler genetik incelemeler etkili bir şekilde tanıya ulaşmayı sağlayabilir.

Anahtar kelimeler: Kreatin kinaz, Duchenne müsküler distrofi, nöromüsküler bulgular, tüm ekzom dizileme, yeni nesil dizileme
Received/Geliș: 20.02.2020

Accepted/Kabul: 06.05.2020

Published Online/Online Yayın: 31.08.2020

Tuğçe Aksu Uzunhan

Sağlık Bilimleri Üniversitesi, Okmeydanı Eğitim ve Araştırma Hastanesi, Çocuk Nöroloji Kliniği, İstanbul - Turkey tugceuzunhan@yahoo.com ORCiD: 0000-0003-0596-2690

B. Ertürk 0000-0002-0348-6267 Sağlık Bilimleri Üniversitesi, Okmeydanı Eğitim ve Arasstırma Hastanesi, Tıbbi Genetik Kliniği, Istanbul, Türkiye

P. Özyavuz Çubuk 0000-0002-8951-7959 Sağlık Bilimleri Üniversitesi, Haseki Eğitim ve Araștırma Hastanesi, Tibbi Genetik Kliniği, istanbul, Türkiye

B. Uyanık 0000-0002-1714-3740 Sağlık Bilimleri Üniversitesi, Bakırköy Dr Sadi Konuk Eğitim ve Araştırma Hastanesi, Tıbbi Genetik Kliniği, İstanbul, Türkiye

A. Ayaz 0000-0001-6930-7148 Medipol Üniversitesi Tıp Fakültesi, Tibbi Genetik Anabilim Dalı, istanbul, Türkiye

O. Akan 0000-0001-8520-670X Sağlık Bilimleri Üniversitesi, Okmeydanı Eğitim ve Araştırma Hastanesi, Nöroloji Kliniği, Istanbul, Türkiye

T.R. Özdemir 0000-0003-4870-6945 Sağlık Bilimleri Üniversitesi, Izmir Tepecik Eğitim ve Araştırma Hastanesi, Genetik Hastalıklar Tanı Merkezi, izmir, Türkiye 


\section{INTRODUCTION}

Neuromuscular disorders are the spectrum of diseases in which the primary abnormality is in the peripheral nervous system, which involves the anterior horn cell, the peripheral nerve, the neuromuscular junction, and the muscle ${ }^{(1)}$. They can appear at any age and may present with positive (cramps, contractures, muscle hypertrophy, and myalgias) and negative (exercise intolerance, fatigue, and muscle atrophy and weakness) symptoms ${ }^{(2,3)}$. Traditional diagnostic tests are electromyography (EMG), nerve conduction studies, and muscle biopsy ${ }^{(4)}$. During the past 20 years, advancements in the field of genetic medicine have changed the diagnostic tests for neuromuscular diseases ${ }^{(4)}$. Even though effective, genetic testing has added another level of complexity to an already complex diagnostic field and has left practitioners uncertain about the indications for invasive procedures such as EMG or muscle biopsy ${ }^{(4)}$. Even with all the common and easily available diagnostic techniques, including genetic tests, some patientsmostly those with the rarer subtypes of neuromuscular disorders-cannot get a definite diagnosis, thus necessitating a more comprehensive genomic approach.

The aim of our study is to investigate patients applied to a pediatric neurology outpatient clinic who exhibited signs and symptoms of neuromuscular disorders, to explore how many of them received a specific diagnosis by genetic testing and how many of them needed next generation sequencing (NGS) as well as to study their clinical history, laboratory investigations, diagnoses, treatment, and consultations.

\section{MATERIALS and METHOD}

Patients aged $0-18$ years admitted to the outpatient pediatric neurology clinic of Okmeydanı Training and Research Hospital between January 2017 and July 2019 with complaints and signs that indicated neuromuscular disorders were included in the study. All patients were recorded so to investigate the number of patients with neuromuscular manifestations. Patients with acute neuromuscular disorders (e.g., acute rhabdomyolysis and Guillain-Barré syndrome), neuropathy or muscle weakness due to chemotherapy treatment and drugs, and spinal cord malformations, as well as patients diagnosed previously in different centers, were excluded from the study. The outpatient pediatric neurology clinic books appointments over telephone or website after referral from a pediatrician. Complaints and signs of neuromuscular disorders included cramps, contractures, muscle hypertrophy, muscle pseudohypertrophy, motor developmental delay, fasciculations, reduced or absent deep tendon reflexes, myotonia, foot drop, myalgias, exercise intolerance, fatigue, fatigability, muscle atrophy, weakness, and hypotonia. Laboratory signs in patients being referred to the pediatric neurology clinic were elevated CK levels that were either symptomatic or asymptomatic. High CK levels were considered to be levels 2-fold higher than our institution's upper normal level of 205 IU/L. Age, date of admittance, complaints, findings on physical examination, medical history, results of laboratory tests, electrophysiological studies, radiological investigations, genetic test, and final diagnosis, treatments, consultations, and compliance with follow-up were retrospectively recorded from the medical data of patients. Fourteen different genetic tests in different combinations were carried out on patients. Patients were grouped into four categories according to their symptoms and CK levels: symptomatic CK elevation, asymptomatic CK elevation, symptomatic patients without CK elevation, and hypotonic infants.

Some diseases or conditions that lack a definite genetic diagnostic tool were described here in detail to explain the diagnostic method. Hypotonia was defined as a state of low muscle tone (the amount of tension or resistance to stretch in a muscle), often involving reduced muscle strength. CK levels were not taken into consideration when making the diagnosis of hypotonia in an infant ${ }^{(5)}$. Chronic inflammatory demyelinating neuropathy (CIDP) was determined according to diagnostic criteria ${ }^{(6)}$. Congenital myasthenic syndrome is characterized by fatigable weakness of the skeletal muscles (e.g., ocular, bulbar, and limb muscles) with onset at or shortly after 
birth or in early childhood. The diagnosis is based on a decremental EMG response of the compound muscle action potential (CMAP) on low-frequency (2-3 Hz) stimulation, a positive response to acetylcholinesterase (AchE) inhibitors, the absence of antiacetylcholine receptor (AChR), and lack of improvement of clinical symptoms with immunosuppressive therapy ${ }^{(7)}$. Diagnosis of hypokalemic periodic paralysis depends on a history of attacks of muscle weakness associated with documented serum potassium levels of $<3.5 \mathrm{mmol} / \mathrm{L}$ during attacks ${ }^{(8)}$. Thirty percent of patients meeting the diagnostic criteria did not have a mutation in CACNA1S or SCN4Agenes. Ethical approval was obtained from the institutional review board, and descriptive statistical analyses (mean, standard deviation, median, first quadrant, third quadrant, frequency, percentage, minimum, maximum) were performed using SPSS 21.0.

\section{RESULTS}

Forty-five patients were identified with symptoms and signs indicating neuromuscular disease. The total number of 7.149 patients admitted to the outpatient pediatric neurology clinic during the study period. The $0.06 \%$. of the patients manifested peripheral nervous system symptoms in the clinic .The mean age of the patients was $67.8 \pm 59.6$ months; the youngest patient was one month old, the oldest was 17 years, and the median age was 43 months. Thirteen (29\%) patients were female, and 32 (71\%) were male. Mean follow-up period was $9.5 \pm 7.3$ months, and the median follow-up was nine months. Patients were followed up between one and 26 months. The most common physical examination finding was Gowers' sign. For all the patients except one with spinal muscular atrophy type I phenotype, CK levels were measured. CK levels were normal in 18 (39\%) patients and increased in 26 (61\%) patients. One patient who was diagnosed as a hypotonic infant had CK levels of $489 \mathrm{IU} / \mathrm{L}$. Median CK level was $3.211 \mathrm{IU} / \mathrm{L}$; (maximum $26.024 \mathrm{IU} / \mathrm{L}$, and minimum $413 \mathrm{IU} / \mathrm{L})$. Eighteen (40\%) patients had symptomatic CK, and seven (16\%) patients asymptomatic CK elevation. While $14(31 \%)$ patients had symptoms wit- hout CK elevation, and six (13\%) patients were hypotonic infants.

Twenty-four (53\%) patients underwent an EMG which demonstrated diffuse myopathic changes in 4, results suggesting myasthenia in two, mild myopathic changes in one, and myotonia in one patient. The EMG of seven patients showed neuropathic changes. A cranial MRI was performed on 13 (30\%) patients, the results of which were normal in 11 patients. Two patients-one without a definite diagnosis and one with congenital myotonic dystrophy-had polymicrogyria and hydrocephaly, respectively. Spinal MRI was performed on nine patients, and only one patient who had chronic inflammatory demyelinating polyneuropathy (CIDP)) had contrast enhancement in the lumbosacral root and fibers. Muscle biopsy was performed on three (7\%) patients and showed only nonspecific myopathic changes. Echocardiographic and electrographic investigations were done for 42 patients. While 40 had normal findings, one patient without a definite diagnosis had arrhythmia, and one patient diagnosed with Ullrich muscular dystrophy had aorta insufficiency and a bicuspid aortic valve. Two patients with CIDP had acellular protein elevation in CSF. Tests for inborn diseases of the metabolism including tandem MS, acylcarnitine profile, urine organic acid analysis, and serum quantitative amino acid and lactate test results were within normal limits in 19 (42\%) patients, and these tests were not ordered for the other 26 patients. Eight (8\%) patients who had proximal muscle weakness and CK elevation were evaluated for Pompe disease with the dry blood spot (DBS) technique for enzymatic analysis, but these patients had normal levels.

Genetic tests in different combinations were carried out on 38 patients. Seven patients did not have any genetic tests performed; two of them were diagnosed with CIDP, and the others did not attend their follow-up appointments regularly. The third patient with CIDP underwent PMP22 MLPA analysis. The most common genetic tests in decreasing order of frequency were dystrophinopathy multiplex polymerase chain reaction (m-PCR) analysis, dystrophinopathy multiplex ligation-dependent probe amplifica- 
Table 1. Features of patients who had a definite diagnosis either by genetic testing or clinically.

\begin{tabular}{|c|c|c|c|c|c|}
\hline $\begin{array}{l}\text { Age } \\
\text { G }\end{array}$ & Symptoms & Physical examination & Family history & $\begin{array}{l}\text { Genetic Test } \\
\text { Genetic Results }\end{array}$ & Definite diagnosis \\
\hline $\begin{array}{l}4 \text { yrs } \\
M\end{array}$ & Cramps & $\begin{array}{l}\text { Gowers sign } \\
\text { Calf muscle } \\
\text { pseudo-hypertrophy }\end{array}$ & No & $\begin{array}{l}\text { Dystrophin MLPA analysis } \\
\text { Hemizygous deletion in } \\
\text { exon } 52 \text { of } \\
\text { dystrophin gene }\end{array}$ & Dystrophynopathy \\
\hline $\begin{array}{l}22 \operatorname{mos} \\
M\end{array}$ & $\begin{array}{l}\text { Cannot } \\
\text { walk }\end{array}$ & $\begin{array}{l}\text { Proximal muscle } \\
\text { weakness }\end{array}$ & $\begin{array}{l}\text { Muscle disease in } \\
\text { two uncles }\end{array}$ & $\begin{array}{l}\text { Dystrophin MLPA analysis } \\
\text { Hemizygous duplication in } \\
\text { exons } 45-50 \text { of dystrophin } \\
\text { gene }\end{array}$ & Dystrophynopathy \\
\hline $\begin{array}{l}39 \operatorname{mos} \\
M\end{array}$ & $\begin{array}{l}\text { Difficulty climbing } \\
\text { stairs }\end{array}$ & $\begin{array}{l}\text { Gowers sign } \\
\text { calf muscle } \\
\text { pseudo-hypertrophy }\end{array}$ & No & $\begin{array}{l}\text { Dystrophin MLPA analysis } \\
\text { Hemizygous deletion in } \\
\text { exon } 49-50 \text { of dystrophin } \\
\text { gene }\end{array}$ & Dystrophynopathy \\
\hline $\begin{array}{l}30 \text { mos } \\
\mathrm{M}\end{array}$ & $\begin{array}{l}\text { Falls during } \\
\text { walking }\end{array}$ & $\begin{array}{l}\text { Gowers sign } \\
\text { calf muscle } \\
\text { pseudo-hypertrophy }\end{array}$ & No & $\begin{array}{l}\text { Dystrophin m-PCR analysis } \\
\text { Hemizygous deletion in } \\
\text { exon 19-43-44 of } \\
\text { dystrophin gene }\end{array}$ & Dystrophynopathy \\
\hline $\begin{array}{l}9 \text { mos } \\
M\end{array}$ & No crawling & No & $\begin{array}{l}\text { Two uncles died of } \\
\text { muscle disease } \\
\text { (Mother carrier of } \\
\text { exon } 12 \text { deletion) }\end{array}$ & $\begin{array}{l}\text { Dystrophin m-PCR analysis } \\
\text { Hemizygous deletion in } \\
\text { exon } 12 \text { of dystrophin } \\
\text { gene }\end{array}$ & Dystrophynopathy \\
\hline $\begin{array}{l}18 \text { mos } \\
M\end{array}$ & $\begin{array}{l}\text { Cannot get up from a } \\
\text { sitting position, } \\
\text { difficulty in language } \\
\text { acquisition }\end{array}$ & $\begin{array}{l}\text { Can not walk } \\
\text { independently }\end{array}$ & No & $\begin{array}{l}\text { Dystrophin m-PCR analysis } \\
\text { Hemizygous deletion in } \\
\text { exon } 45-67 \text { of dystrophin } \\
\text { gene }\end{array}$ & Dystrophynopathy \\
\hline $\begin{array}{l}36 \text { mos } \\
M\end{array}$ & CK elevation & Asymptomatic & $\begin{array}{l}\text { No } \\
\text { (Mother carrier of } \\
\text { exon 10-29 deletion) }\end{array}$ & $\begin{array}{l}\text { Dystrophin m-PCR analysis } \\
\text { Hemizygous deletion in } \\
\text { exon } 10-29 \text { of dystrophin } \\
\text { gene }\end{array}$ & Dystrophynopathy \\
\hline $\begin{array}{l}34 \text { mos } \\
M\end{array}$ & $\begin{array}{l}\text { Difficulty climbing } \\
\text { stairs }\end{array}$ & Gowers sign & No & $\begin{array}{l}\text { Dystrophin m-PCR analysis } \\
\text { Hemizygous deletion in } \\
\text { exon 3-44 of dystrophin } \\
\text { gene }\end{array}$ & Dystrophynopathy \\
\hline $\begin{array}{l}9 \text { mos } \\
M\end{array}$ & No & Normal & $\begin{array}{l}\text { Yes } \\
\text { (A so-called DMD in } \\
\text { brother not genetically } \\
\text { proven) }\end{array}$ & $\begin{array}{l}\text { Clinical Exome Sequencing } \\
\text { Hemizygous mutation in } \\
\text { Dystrophin gene } \\
\text { c.4669_4670del } \\
\text { (p.GIn1557Valfs*10) }\end{array}$ & Dystrophynopathy \\
\hline $\begin{array}{l}20 \text { mos } \\
\mathrm{F}\end{array}$ & Can not walk & Gowers sign & $\begin{array}{l}\text { Uncle with muscle } \\
\text { disease (Mother } \\
\text { carrier of exon } 12 \\
\text { deletion) }\end{array}$ & $\begin{array}{l}\text { Dystrophin MLPA analysis } \\
\text { Carrier of heterozygous } \\
\text { deletion in exon } 12 \text { of } \\
\text { dystrophin gene }\end{array}$ & $\begin{array}{l}\text { Dystrophynopathy } \\
\text { (symptomatic carrier) }\end{array}$ \\
\hline $\begin{array}{l}30 \text { mos } \\
\mathrm{F}\end{array}$ & $\begin{array}{l}\text { Elevated liver } \\
\text { enzymes }\end{array}$ & Normal & $\begin{array}{l}\text { Father has difficulty } \\
\text { climbing stairs (Father } \\
\text { has hemizygous deletion } \\
\text { in exons } 45-55 \text { of } \\
\text { dystrophin gene) }\end{array}$ & $\begin{array}{l}\text { Dystrophin MLPA analysis } \\
\text { Carrier of heterozygous } \\
\text { deletion in exons } 45-55 \text { of } \\
\text { dystrophin gene }\end{array}$ & $\begin{array}{l}\text { Dystrophynopathy } \\
\text { (asymptomatic carrier) }\end{array}$ \\
\hline $\begin{array}{l}10 \text { yrs } \\
M\end{array}$ & Hand shakiness & $\begin{array}{l}\text { Tremor } \\
\text { Gowers sign Proximal } \\
\text { muscle weakness }\end{array}$ & No & $\begin{array}{l}\text { SMN1 MLPA analysis } \\
\text { Homozygous deletion in } \\
\text { exons } 7-8 \text { of SMN1 gene }\end{array}$ & $\begin{array}{l}\text { Spinal muscular } \\
\text { atrophy type III }\end{array}$ \\
\hline $\begin{array}{l}1,5 \text { mos } \\
F\end{array}$ & Floppiness & $\begin{array}{l}\text { Lost DTR } \\
\text { Tongue fasciculations } \\
\text { No head control } \\
\text { Good social interaction }\end{array}$ & $\begin{array}{l}\text { A sibling as floppy as } \\
\text { her died at three } \\
\text { months without a } \\
\text { definite diagnosis }\end{array}$ & $\begin{array}{l}\text { SMN1 MLPA analysis } \\
\text { Homozygous deletion in } \\
\text { exons 7-8 of SMN1 gene }\end{array}$ & $\begin{array}{l}\text { Spinal muscular } \\
\text { atrophy type I }\end{array}$ \\
\hline
\end{tabular}


T. Aksu Uzunhan et al, Evaluation of Laboratory Findings, Clinical Features and Rates of Diagnosis of Patients Admitted to Outpatient Clinic of Pediatric Neurology with Neuromuscular Manifestations

Table 1. (continuation)

\begin{tabular}{|c|c|c|c|c|c|}
\hline $\begin{array}{l}7 \text { mos } \\
\mathrm{M}\end{array}$ & No head control & $\begin{array}{l}\text { No DTR } \\
\text { Tongue fasciculations } \\
\text { Funnel chest } \\
\text { Good social interaction }\end{array}$ & No & $\begin{array}{l}\text { SMN1 MLPA analysis } \\
\text { Homozygous deletion in } \\
\text { exons 7-8 of SMN1 gene }\end{array}$ & $\begin{array}{l}\text { Spinal muscular } \\
\text { atrophy type I }\end{array}$ \\
\hline $\begin{array}{l}13 \text { yrs } \\
M\end{array}$ & $\begin{array}{l}\text { Weakness and } \\
\text { numbness in legs }\end{array}$ & $\begin{array}{l}\text { Dorsiflexion of right } \\
\text { thumb and four other } \\
\text { fingers is weak. } \\
\text { Dorsiflexion of right } \\
\text { foot is } 4 / 5 \text {. No Achilles } \\
\text { DTR on the right. }\end{array}$ & No & $\begin{array}{l}\text { PMP22 MLPA analysis } \\
\text { Heterozygous deletion in } \\
\text { PMP22 gene }\end{array}$ & $\begin{array}{l}\text { Hereditary neuropathy } \\
\text { with liability to } \\
\text { pressure palsies }\end{array}$ \\
\hline $\begin{array}{l}6.5 \text { yrs } \\
F\end{array}$ & $\begin{array}{l}\text { Frequent falls, } \\
\text { difficulty walking }\end{array}$ & Myotonia & No & $\begin{array}{l}\text { CLCN1 sequence analysis } \\
\text { Homozygous mutation in } \\
\text { CLCN1 gene } \\
\text { c.1886T>C (p. Leu629Pro) }\end{array}$ & $\begin{array}{l}\text { Autosomal recessive } \\
\text { myotonia congenita }\end{array}$ \\
\hline $\begin{array}{l}7 \text { mos } \\
\mathrm{F}\end{array}$ & $\begin{array}{l}\text { Floppiness, referral } \\
\text { for hydrocephalus }\end{array}$ & $\begin{array}{l}\text { Hypotonia, no head } \\
\text { control }\end{array}$ & $\begin{array}{l}\text { Weakness in mother } \\
\text { ( } 5 \text { CTG/>50 CTG } \\
\text { repeats in DMPK gene) }\end{array}$ & $\begin{array}{l}\text { DMPK gene molecular } \\
\text { testing } \\
5 \text { CTG/>50 CTG repeats in } \\
\text { DMPK gene }\end{array}$ & $\begin{array}{l}\text { Congenital myotonic } \\
\text { dystrophy }\end{array}$ \\
\hline $\begin{array}{l}6.5 \text { yrs } \\
M\end{array}$ & No & Normal & No & $\begin{array}{l}\text { LGMD panel testing } \\
\text { Homozygous mutation in } \\
\text { CAPN3 gene } \\
\text { c.1303G>A (p.Glu435Lys) }\end{array}$ & $\begin{array}{l}\text { Calpainopathy } \\
\text { (muscular dystrophy, } \\
\text { limb-girdle, autosomal } \\
\text { recessive } 1\end{array}$ \\
\hline $\begin{array}{l}3.5 \text { yrs } \\
M\end{array}$ & $\begin{array}{l}\text { Difficulty climbing } \\
\text { stairs }\end{array}$ & $\begin{array}{l}\text { Gowers sign } \\
\text { Tip toe walking }\end{array}$ & No & $\begin{array}{l}\text { WES } \\
\text { Compound heterozygous } \\
\text { mutations in COL6A3 gene } \\
\text { c. } 6309+3 A>T \\
\text { c. } 6299 C>G\end{array}$ & $\begin{array}{l}\text { Ullrich muscular } \\
\text { dystrophy I }\end{array}$ \\
\hline $\begin{array}{l}13 \text { yrs } \\
M\end{array}$ & Attacks of paralysis & $\begin{array}{l}\text { Normal other than } \\
\text { attacks }\end{array}$ & No & $\begin{array}{l}\text { WES } \\
\text { Heterozygous mutation in } \\
\text { CACNA1S gene } \\
\text { p.V876E }\end{array}$ & $\begin{array}{l}\text { Hypokalemic periodic } \\
\text { paralysis, type I }\end{array}$ \\
\hline $\begin{array}{l}9,5 \text { yrs } \\
M\end{array}$ & $\begin{array}{l}\text { Difficulty climbing } \\
\text { stairs }\end{array}$ & Gowers sign & No & $\begin{array}{l}\text { Clinical Exome Sequencing } \\
\text { Heterozygous mutation in } \\
\text { KIF1A gene } \\
\text { c. } 761 \mathrm{G}>\text { A p.(Arg254GIn) }\end{array}$ & $\begin{array}{l}\text { Mental retardation, } \\
\text { autosomal dominant } 9\end{array}$ \\
\hline $\begin{array}{l}15 \text { yrs } \\
M\end{array}$ & Frequent falls & $\begin{array}{l}\text { No DTR } \\
\text { Foot drop }\end{array}$ & No & No genetic testing available & CIDP \\
\hline $\begin{array}{l}2.5 \text { yrs } \\
M\end{array}$ & Difficulty walking & $\begin{array}{l}\text { Albinism } \\
\text { Proximal muscle } \\
\text { weakness }\end{array}$ & No & No genetic testing available & CIDP \\
\hline $\begin{array}{l}12.5 \text { yrs } \\
M\end{array}$ & $\begin{array}{l}\text { Weakness in legs and } \\
\text { hands }\end{array}$ & $\begin{array}{l}\text { Gowers sign } \\
\text { Distal weakness in } \\
\text { hands }\end{array}$ & No & No genetic testing available & CIDP \\
\hline $\begin{array}{l}17 \text { yrs } \\
M\end{array}$ & Attacks of paralysis & $\begin{array}{l}\text { Normal other than } \\
\text { attacks }\end{array}$ & $\begin{array}{l}\text { Father with hypokalemic } \\
\text { periodic paralysis }\end{array}$ & No results yet & $\begin{array}{l}\text { Hypokalemic periodic } \\
\text { paralysis }\end{array}$ \\
\hline $\begin{array}{l}8.5 \text { yrs } \\
M\end{array}$ & $\begin{array}{l}\text { Difficulty } \\
\text { lying down at night }\end{array}$ & $\begin{array}{l}\text { Ptosis } \\
\text { External ophtalmoplegia } \\
\text { Fatigability }\end{array}$ & No & No results yet & $\begin{array}{l}\text { Congenital myasthenic } \\
\text { syndrome }\end{array}$ \\
\hline
\end{tabular}

CIDP: chronic inflammatory demyelinating polyneuropathy, CK: creatine kinase, DMD: Duchenne muscular dystrophy, DTR: deep tendon reflexes, F: Female, G: Gender, LGMD: limb girdle muscular dystrophy, M: Male, MLPA: multiplex ligand probe amplification, mos: months, $m-P C R$ : multiplex polymerase chain reaction, WES: whole exome sequencing, yrs: years 
Table 2. Specific diagnoses according to groups.

\begin{tabular}{|c|c|c|c|c|}
\hline & \multicolumn{4}{|c|}{ Groups (Number of Patients) } \\
\hline & $\begin{array}{l}\text { Symptomatic CK } \\
\text { elevation (18) }\end{array}$ & $\begin{array}{l}\text { Asymptomatic CK } \\
\text { elevation (7) }\end{array}$ & $\begin{array}{l}\text { Having symptoms without CK } \\
\text { elevation (14) }\end{array}$ & Hypotonic infant (6) \\
\hline $\begin{array}{l}\text { Specific diagnoses } \\
\text { (Number of Patients) }\end{array}$ & $\begin{array}{l}\text { Unknown (10) } \\
\text { Dystrophinopathy (7) } \\
\text { Spinal muscular } \\
\text { atrophy type III (1) }\end{array}$ & $\begin{array}{l}\text { Unknown (3) } \\
\text { Dystrophinopathy (2) } \\
\text { Dystrophinopathy } \\
\text { asymptomatic carrier (1) } \\
\text { Calpainopathy (1) }\end{array}$ & $\begin{array}{l}\text { CIDP (3) } \\
\text { Unknown (3) } \\
\text { Dystrophinopathy symptomatic } \\
\text { carrier (1) } \\
\text { Hypokalemic periodic paralysis (2) } \\
\text { HNPP (1) } \\
\text { Congenital myasthenic syndrome (1) } \\
\text { Mental retardation, autosomal } \\
\text { dominant } 9 \text { (1) } \\
\text { AR myotonia congenita (1) } \\
\text { Ullrich muscular dystrophy (1) }\end{array}$ & $\begin{array}{l}\text { Spinal muscular } \\
\text { atrophy type I (2) } \\
\text { Unknown (2) } \\
\text { Congenital myotonic } \\
\text { dystrophy (1) } \\
\text { Unknown (1) }\end{array}$ \\
\hline
\end{tabular}

CK: creatine kinase, CIDP: chronic inflammatory demyelinating polyneuropathy, HNPP: hereditary neuropathy with liability to pressure palsies, AR: autosomal recessive

tion (MLPA) analysis, and SMN1 MLPA analysis. Patients were tested 0-4 times to reach a final diagnosis. Seven patients underwent NGS (targeted panels, clinical exome sequencing, and WES). Final diagnoses of dystrophinopathy due to a point mutation, hypokalemic periodic paralysis I, Ullrich muscular dystrophy I, autosomal dominant mental retardation 9, and calpainopathy were confirmed in five patients. A nine-and-a-half-year-old patient with Gowers' sign underwent clinical exome sequencing; and a heterozygous KIF1A mutation was detected with resultant diagnosis of mental retardation, autosomal dominant 9. A patient with known hypokalemic periodic paralysis had his genetic mutation defined. A patient with neuropathy awaiting PMP22 MLPA analysis later turned out to have CIDP. Twentysix (58\%) patients received a definite diagnosis, while 19 (42\%) were not definitively diagnosed. The diagnosis of twenty-one patients (44\%) out of 26 (58\%) was confirmed with genetic testing. While five patients diagnosed without a genetic test including three patients with CIDP, one patient with hypokalemic periodic paralysis, and one patient with congenital myasthenic syndrome. None of the undiagnosed patients underwent NGS. All patients who were diagnosed clinically and genetically are shown in Table 1. The most common diagnosis was dystrophinopathy. Definite diagnoses in categories according to clinical features and CK levels are presented in Table 2.

Acetazolamide at a dose of $20 \mathrm{mg} / \mathrm{kg}$ was started for patients with hypokalemic periodic paralysis, intravenous immunoglobulin $1 \mathrm{~g} / \mathrm{kg}$ was given once every three weeks for CIDP patients, and carbamazepine and mexiletine treatments were given for patients with autosomal recessive myotonia congenita. The patient with congenital myasthenic syndrome was treated with pyridostigmine and tracheostomy. Some precautions to prevent pressure palsies were taken for the patient who had HNPP. No patient with dystrophinopathy was started with steroids because of their young age. All patients benefited from treatment except the patient with autosomal recessive myotonia congenita and one patient with hypokalemic periodic paralysis, who could not be evaluated because he did not come to his appointments. Fourteen (31\%) patients had comorbidities, most commonly delays in language and social development, while 31 (69\%) patients did not have any. A twelve-month-old patient with ptosis, external ophthalmoplegia, and fasciculations died without any diagnosis due to a presumed apneic attack at home. Patients most commonly consulted with the department of physical rehabilitation and secondly with department of pediatric metabolism. Twenty-five (55\%) patients regularly visited our outpatient clinic, 
T. Aksu Uzunhan et al, Evaluation of Laboratory Findings, Clinical Features and Rates of Diagnosis of Patients Admitted to Outpatient Clinic of Pediatric Neurology with Neuromuscular Manifestations

while 20 (45\%) patients did not attend their appointments regularly. Of the nineteen undiagnosed patients, $10(53 \%)$ patients regularly attended the outpatient clinic, and nine (47\%) patients did not.

\section{DISCUSSION}

Neuromuscular disorders cover a large variety of diseases of the peripheral nervous system, and in children and adolescents most cases are due to hereditary causes. However, acquired diseases that are mostly inflammatory with treatable etiologies should not be missed. Since the identification of dystrophin mutations in Duchenne muscular dystrophy in 1985, an increasing number of causative genes has been identified, which has led to accurate diagnosis, genetic counseling, and pathophysiological understanding of pediatric neuromuscular disorders ${ }^{(9)}$. We described patients with CK elevation and symptomatology suggesting neuromuscular disorders coming from a pediatric neurology outpatient clinic and analyzed the pathway leading to the diagnosis. Patients with CK elevation in our study were both symptomatic and asymptomatic, although more cases were symptomatic. The most common cause was dystrophinopathy in both groups, compatible with the fact that the most common childhood neuromuscular disease is Duchenne muscular dystrophy, a dystrophinopathy with an incidence of $1 / 3,500-1 / 5,000$ in male children ${ }^{(10)}$. A recent study presented a spectrum of hyperCKemia according to the extent of CK elevation; the most common diagnosis being dystrophinopathy within all three groups. There was also hyperCKemia due to neurogenic disorders like spinal muscular atrophy ${ }^{(11)}$ as in our patient with spinal muscular atrophy type III, who had elevated CK levels.

All of our dystrophinopathy patients were diagnosed genetically using m-PCR, MLPA and, in one patient, clinical exome sequencing to detect point mutations of dystrophin. Before the widespread use of dystrophin m-PCR analysis, muscle biopsy was the sole diagnostic technique for dystrophinopathies. In a study by Ardıçlı et al., muscle biopsy was performed after a negative deletion/duplication test for dystrophinopathies in $42 \%$ of the patients, but the percentage of patients who underwent diagnostic muscle biopsy declined after 2011, and no patient underwent this test in the last two years of their study ${ }^{(12)}$. Still, muscle biopsy gives invaluable clues in identifying pediatric neuromuscular disorders like limb girdle muscular dystrophies and the congenital myopathies described by Diniz et al ${ }^{(13)}$.

The most common neuromuscular disorders reported after dystrophinopathies are myotonic dystrophy and spinal muscular atrophy ${ }^{(14,15)}$. Our patient population includes one congenital myotonic dystrophy patient whose mother got a diagnosis of myotonic dystrophy after her daughter's diagnosis and three patients with spinal muscular atrophy. We did not have a patient with classical myotonic dystrophy type I. This could be due to several factors; for example, the age of onset is $10-30$ years, and since clinical signs like weakness may not be prominent in early childhood, myotonia could be missed during a routine neurological examination (16). Diseases like spinal muscular atrophy are more common in Turkey than are globally reported due to consanguineous marriages, but myotonic dystrophy has an autosomal dominant inheritance pattern.

Late-onset Pompe disease is an important inherited neuromuscular disorder because it is treatable. Ünver et al. reported three cases of late -onset Pompe disease out of 37 pediatric patients with limb girdle weakness and 35 patients with nonspecific hyperCKemia diagnosed by a DBS test and genetically confirmed. They started enzyme replacement treatment following the guidelines of the American Association of Neuromuscular \& Electrodiagnostic Medicine (AANEM) ${ }^{(17)}$. Chronic inflammatory demyelinating neuropathy, another treatable but immune-mediated disease of the peripheral nervous system and a common diagnosis in our cohort, lacks a reliable biological marker, leading to challenges in diagnosis, follow-up, and treatment ${ }^{(6)}$. CharcotMarie-Tooth (CMT) disease is a differential diagnosis of CIDP. Analysis for PMP22 gene duplication in CMT1A, the most common form of CMT, was performed in one of our CIDP patients. As CMT has no cure, treatment for CIDP should be considered as 
early as possible in children who meet the diagnostic criteria with neuropathic symptoms ${ }^{(6)}$.

The most favorable diagnostic approach to a patient suspected to have an inherited neuromuscular disorder is to determine an initial diagnosis through history, physical examination, and CK levels and then to request a targeted genetic analysis according to the initial diagnosis. For the initial diagnosis of dystrophinopathy, the diagnostic algorithm is precisely defined, but sometimes the clinical signs, symptoms, and CK levels do not suggest a diagnosis, limiting the diagnostic rate using phenotype-driven genetic testing. Thus, for such patients, early application of NGS such as WES is recommended in neuromuscular disorders as they are likely to yield a high diagnostic rate ${ }^{(18,19)}$. We had seven patients who underwent NGS, and five of them received a diagnosis. In a U.K. study, exome sequencing was compared with sequential gene testing for limb girdle muscular dystrophy. Those patients selected for WES had undergone more extensive prior testing than those undergoing standard genetic testing. They concluded that WES achieved a higher rate of diagnosis than standard testing even in a cohort of extensively investigated patients ${ }^{(18)}$.

Vill et al. retrospectively analysed 98 patients with early onset myopathies. Final diagnosis was possible for 63 (64\%) out of 98 patients in a single neuromuscular referral center. We were able to diagnose more than half of our patients over a two-anda-half-year period genetically and clinically using high throughput genetic methods like NGS when necessary in a single center of pediatric neurology, which schedules appointments by telephone or online after referral from a pediatrician for general purposes in the field of pediatric neurology. The limitations of our study include its retrospective nature, our inability to perform NGS on all undiagnosed patients as NGS is not still extensively used, and our inability to give incidence/prevalence rates by county or city. Our one patient with presumed neuromuscular disorder was diagnosed with mental retardation, autosomal dominant type 9, a KIF1A mutation. Sometimes, diseases like connective tissue disorders and metabolic disorders can mimic myopathy ${ }^{(20,21)}$.
Neuromuscular disorders can present with asymptomatic or symptomatic CK elevation and without any CK elevation. According to medical history, physical examination, and CK levels, treatable etiologies of neuromuscular diseases should be considered first for every patient. Despite all efforts, a group of patients still may not get a diagnosis. Monitoring patients, even undiagnosed patients on a regular basis, could be useful as new diagnostic approaches and treatment opportunities may be possible in the future.

Ethics Committee Approval: T. C. Approval was obtained from the Istanbul Governorship Provincial Health Directorate Okmeydanı Training and Research Hospital Ethics Committee (27.08.2019/1418).

Conflict of Interest: The authors declared no potential conflicts of interest with respect to the research, authorship and/or publication of this article.

Funding: The authors received no financial support for the research, authorship, and/or publication of this article.

Informed Consents: Since our study was retrospective, informed consent was not taken.

\section{REFERENCES}

1. Dowling JJ, D Gonorazky H, Cohn RD, Campbell C. Treating pediatric neuromuscular disorders: The future is now. Am J Med Genet Part A. 2018;176(4):804-41. https://doi.org/10.1002/ajmg.a.38418

2. Pagola-Lorz I, Vicente E, Ibáñez B, Torné L, Elizalde-Beiras I, Garcia-Solaesa V, et al. Epidemiological study and genetic characterization of inherited muscle diseases in a northern Spanish region. Orphanet J Rare Dis. 2019;14(1). https://doi.org/10.1186/s13023-019-1227-x

3. Barohn RJ, Dimachkie MM, Jackson CE. A pattern recognition approach to patients with a suspected myopathy. Neurol Clin. 2014;32(3):569-93. https://doi.org/10.1016/j.ncl.2014.04.008

4. Darras BT, Jones HR. Diagnosis of pediatric neuromuscular disorders in the era of DNA analysis. Pediatr Neurol. 2000;23(4):289-300.

https://doi.org/10.1016/S0887-8994(00)00202-2

5. Leyenaar J, Camfield P, Camfield C. A schematic approach to hypotonia in infancy. Paediatr Child Health. 2005;10(7):397400.

http://www.ncbi.nlm.nih.gov/pubmed/19668647 https://doi.org/10.1093/pch/10.7.397

6. Haliloğlu G, Yüksel D, Temoçin CM, Topaloğlu H. Challenges in pediatric chronic inflammatory demyelinating polyneuropathy. Neuromuscul Disord. 2016;26(12):817-24. https://doi.org/10.1016/j.nmd.2016.09.016 
7. Engel AG, Shen XM, Selcen D, Sine SM. Congenital myasthenic syndromes: Pathogenesis, diagnosis, and treatment [Internet]. Vol. 14, The Lancet Neurology. Lancet Publishing Group; 2015:420-34.

http://www.ncbi.nlm.nih.gov/pubmed/25792100 https://doi.org/10.1016/S1474-4422(14)70201-7

8. Sansone V, Meola G, Links TP, Panzeri M, Rose MR. Treatment for periodic paralysis. Cochrane Database Syst Rev. 2008;(1).

https://doi.org/10.1002/14651858.CD005045.pub2

9. Korinthenberg R. Neuromuscular disorders in children and adolescents. Neuropediatrics. 2017;48(4):209-10. https://doi.org/10.1055/s-0037-1603980

10. Wei Y, McCormick A, MacKenzie A, O’Ferrall E, Venance S, Mah JK, et al. The Canadian Neuromuscular Disease Registry: Connecting patients to national and international research opportunities. Paediatr Child Heal. 2018;23(1):20-6. https://doi.org/10.1093/pch/pxx125

11. Al-Ghamdi F, Darras BT, Ghosh PS. Spectrum of neuromuscular disorders with hyperCKemia rrom a tertiary care pediatric neuromuscular center. J Child Neurol. 2018;33(6):389-96. http://www.ncbi.nlm.nih.gov/pubmed/29577809 https://doi.org/10.1177/0883073818758455

12. Ardıçlı D, Haliloğlu G, Alikaşifoğlu M, Topaloğlu H. Diagnostic pathway to nonsense mutation dystrophinopathy: A tertiarycenter, retrospective experience. Neuropediatrics. 2019;50(1):41-5. http://www.ncbi.nlm.nih.gov/pubmed/30453357 https://doi.org/10.1055/s-0038-1675626

13. Diniz G, Tosun Yildirim H, Unalp A, Barutcuoglu M, Guzel O, Polat $M$, et al. The evaluation of muscle biopsy findings in children with neuromuscular disorders. J Dr Behcet Uz Child Hosp. 2013;2(2):62-7. https://doi.org/10.5222/buchd.2012.062

14. Campbell C, Levin S, Siu VM, Venance S, Jacob P. Congenital myotonic dystrophy: Canadian population-based surveillance study. J Pediatr. 2013;163(1):120-5.e1-3. http://www.ncbi.nlm.nih.gov/pubmed/23415617 https://doi.org/10.1016/j.jpeds.2012.12.070

15. D’Amico A, Mercuri E, Tiziano FD, Bertini E. Spinal muscular atrophy. Orphanet J Rare Dis. 2011;6:71. http://www.ncbi.nlm.nih.gov/pubmed/22047105 https://doi.org/10.1186/1750-1172-6-71

16. Bird T. Myotonic Dystrophy Type 1. In: Adam M, Ardinger $H$, Pagon R, Wallace S, Bean L, Stephens K, et al., editors. GeneReviews ${ }^{\circledR}$. Seattle;

17. Cupler EJ, Berger KI, Leshner RT, Wolfe GI, Han JJ, Barohn RJ, et al. Consensus treatment recommendations for late-onset Pompe disease. Muscle Nerve. 2012;45(3):319-33. http://www.ncbi.nlm.nih.gov/pubmed/22173792 https://doi.org/10.1002/mus.22329

18. Harris E, Topf A, Barresi R, Hudson J, Powell H, Tellez J, et al. Exome sequences versus sequential gene testing in the UK: Highly specialised Service for Limb Girdle. Muscular Dystrophy. Orphanet J Rare Dis. 2017;12(1):151. http://www.ncbi.nIm.nih.gov/pubmed/28877744 https://doi.org/10.1186/s13023-017-0699-9

19. Westra D, Schouten MI, Stunnenberg BC, Kusters B, Saris CGJ, Erasmus CE, et al. Panel-based exome sequencing for neuromuscular disorders as a diagnostic service. J Neuromuscul Dis. 2019;6(2):241-58. http://www.ncbi.nlm.nih.gov/pubmed/31127727 https://doi.org/10.3233/JND-180376

20. Dusanic M, Dekomien G, Lücke T, Vorgerd M, Weis J, Epplen $\mathrm{JT}$, et al. Novel nonsense mutation in SLC39A13 initially presenting as myopathy: Case report and review of the literature. Mol Syndromol. 2018;9(2):100-9.

http://www.ncbi.nlm.nih.gov/pubmed/29593477 https://doi.org/10.1159/000485881

21. Nimmo GAM, Ejaz R, Cordeiro D, Kannu P, MercimekAndrews S. Riboflavin transporter deficiency mimicking mitochondrial myopathy caused by complex II deficiency. Am J Med Genet A. 2018;176(2):399-403. http://www.ncbi.nlm.nih.gov/pubmed/29193829 https://doi.org/10.1002/ajmg.a.38530 